\title{
Política de visado y regulación de lasfronteras. Un análisis desde la movilidad de haitianos en Sudamérica
}

\author{
Carina I. Trabalón \\ Universidad Nacional de Villa María, Córdoba, Argentina. \\ Email: carinatrabalon522@gmail.com
}

Resumen: El presente trabajo busca abordar el nexo movilidad, fronteras y seguridad con relación al recorrido histórico reciente de la migración haitiana en Sudamérica, destacando para ello el papel que desempeñan los Estados en los procesos securitización, ilegalización y criminalización de las migraciones internacionales. Los objetivos se dirigen aprofundizar el estudiode las condiciones de emergencia, efectos prácticos yfundamentosa través de los cuales se implementa la política de visado en Brasil, Chile y, con especial énfasis, en el marco nacional argentino.En este sentido, se adoptó una metodología cualitativa que incluyóel análisis de documentos tales como informes estadísticos, normativas y notas periodísticas que remiten, de manera situada, a la política de visado dirigida a personas de nacionalidad haitiana en cada uno de los países mencionados.Elartículo revela cómociertos grupos nacionales se incorporan a la agenda de seguridad por medio de prácticas estatales selectivas que, bajo similares argumentos securitarios y humanitarios de control, posicinoan al visado como mecanismo fundamental para la regulación de los movimientos migratoriosa través de las fronteras. seguridad.

Palabras clave: Sudamérica, migración haitiana, visado, fronteras,

\section{Visa policy and regulation of borders. An analysis from the mobility of Haitians in South America}

\begin{abstract}
This paper seeks to address the nexus mobility, borders and security in relation to the recent historical journey of Haitian migration in South America, highlighting for this the role played by states in the processes of securitization, illegalization and criminalization of international migration. The objectives are aimed at deepening the study of the emergency conditions, practical effects and fundamentals through which the visa policy is implemented in Brazil, Chile and, with special emphasis, in the Argentine national framework. In this sense, a qualitative methodology was adopted that included the analysis of documentary statistical reports, regulations and journalistic notes that refer, in a localized manner, to the visa policy addressed to persons of Haitian nationality in each of the mentioned countries. The study reveals how certain national groups are incorporated into the security agenda through selective state practices that, under similar security and humanitarian control arguments, position the visa as a funda-
\end{abstract}


mental mechanism for the regulation of migratory movements across borders.

Keywords: South America, Haitian migration, visa, borders, security.

\section{Política de vistos e regulação de fronteiras. Uma análise da mobilidade dos haitianos na América do Sul}

Resumo: No presente trabalho, procuramos abordar as nexo de mobilidade, fronteiras e segurança em relação à recente turnê histórica da migração haitiana na América do Sul, com destaque para isso o papel dos Estados na securitização processos, proibindo e criminalização da migração internacional. Os objetivos visam aprofundar o estudo das condições de emergência, efeitos práticos e fundamentos através dos quais a política de vistos é implementada no Brasil, Chile e, com especial ênfase, no quadro nacional argentino. Nesse sentido, adotou-se uma metodologia qualitativa que incluiu a análise documental de relatórios estatísticos, regulamentos e notas jornalísticas que se referem, de maneira localizada, à política de vistos dirigida a pessoas de nacionalidade haitiana em cada um dos países mencionados. O estudo revela como certos grupos nacionais são incorporados à agenda de segurança por meio de práticas seletivas do Estado que, baixosemelhantes argumentos de segurança e humanitários, posicionam o visto como um mecanismo fundamental para a regulação dos movimentos migratórios através das fronteiras.

Palavras-chave: América do Sul, migração haitiana, vistos, fronteiras, segurança.

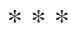

\section{Introducción}

En el presente trabajo se proponeanalizarla política de visado dirigida a regular la movilidad de haitianos en la región, con el objetivo de inscribir la aparición de estas y otras formas de movilidadhacia y en Argentina,en el marco de procesos más generales asociados al régimen global de control (Düvell, 2003) y ciertasparticularidadesreferidas a la “gestión” de las fronteras en el espacio sudamericano.En este marco, la movilidad reciente de haitianos en Sudamérica, desde 2010 al presente, se encuentra atravesada por diversos procesos de transformación yun reacomodamiento constante de sus redes migratorias. Esto puede verse, en parte, como resultado de la implementación de diferentes medidas de control migratorioy fronterizo (visados, rechazos en frontera, preavisos de viaje, planes de regularización migratoria, planes de retorno "humanitario", entre otros), las vicisitudes propias de las economías latinoamericanas y un clima político desfavorable propiciado por el avance del neoliberalismo y el recrudecimiento de los discursos xenófobos y racistas en la región. Estas circunstancias se han traducido, entre otras cosas, en una gran inestabilidad de los proyectos migratorios de haitianos que, frente a situaciones adversas de distinta índole, ven restringida su movilidad, ingreso y permanencia en Argentina y otros países sudamericanos.

En este escenario, en primer lugar, se plantean posibles cruces teóri- 
cos para abordar el nexo movilidad, fronteras y seguridad con relación al recorrido histórico reciente de la migración haitiana en América del Sur. En segundo lugar, se analizan las condiciones de emergencia y los efectos prácticos de la política de visado implementada en Brasil, Chile y, en particular, en el marco nacional argentino. Por último, se examinan los fundamentos securitarios y humanitarios de estas prácticas de control y sus posibles implicancias para la movilidad de haitianos en la región.

Se considera que la referencia constante auna migración “ordenada, segura y regular” no es una formula vacía sino que supone una manera específica de comprender y actuar sobre las migraciones internacionales, bajo un modelo de“gobernanza"que propone la "gestión” de las poblaciones de manera eficiente, según lógicas de costo-beneficio y/o ventajasdesventajas (Domenech, 2013). En este sentido, el análisis de las fronteras a través de la política de visado, dirigida a este grupo nacional en particular, revelauna convergencia,en diferentes países de la región, de discursos y prácticas de control migratorio y fronterizo en la que los migrantes haitianos (como otros grupos) resultan con un "saldo negativo" enlaecuación que el paradigma de la gobernanza de las migraciones impulsa. Asimismo, a pesar de la proliferación de clasificaciones estatales vinculadas a las "nuevas amenazas internacionales" (terrorismo, narcotráfico, trata y tráfico ilícito de personas, etc.), el visado como mecanismo regulación pone en evidenciala persistencia del origen nacional como principio de selección fundamental de las poblaciones móviles. De este modo, se sostendrá que, más allá de la retórica humanitarista y de las particularidades de los marcos nacionales, los procesos analizados expresan cómo, en última instancia, la "seguridad del Estado" prevalece por sobre la "seguridad de los migrantes".

Con relación a la metodología, el artículo se elaboró a partir de un enfoque cualitativo que incluyó el análisis documental de informes estadísticos, notas periodísticas, comunicados de prensa y normativas (resoluciones, disposiciones, memorandos, entre otros)que remiten alas prácticas de rechazos en frontera y, en particular, a la implementación de los visados en Brasil, Argentina y Chilea partir de los movimientos migratorios de haitianos que acontecen en la región desde 2010 al presente.

\section{Migración haitiana en Sudamérica: movilidad, seguridady fronteras}

A nivel mundial, entre los países y/o regiones con mayor número de haitianos y haitianas se identifica a Estados Unidos, Canadá, Francia, República Dominicana, Cuba y América del Sur (Nieto, 2014). Los estudios consultados sobre migración haitiana en distintos países de la región ${ }^{1}$ coinciden en señalar que,a partir del terremoto del 2010 cobra impulso un nuevo flujo migratorio de haitianos hacia Sudamérica, principalmente hacia Brasil, seguido de Chile y, en menor medida, hacia la Argentina.Al mismo tiempo, Ecuador y Perú son considerados como los países de mayor tránsi- 
to de haitianos en la región, aunque también, de manera más reciente, se ha señalado a Ecuador como “destino” (Cárdenas, 2015).

En este escenario, el complejo entramado de prácticas estatales de control y regulación de la movilidad humana a escala regional excede ampliamente los límites de este análisis. Por ello,sin intención de trazar un esquema comparativo entre marcos nacionales y sus respectivas normativas en materia migratoria,se busca señalar el desarrollo ciertas prácticas estatales de control con la finalidad de abrir nuevas interrogantes sobre las trayectorias de migrantes haitianos en torno al control y las fronteras a escala nacional y regional. En particular, interesa abordarla política de visado implementada en Brasil, entre 2012 y 2013,y en Chiley Argentinadurante el 2018.

Se considera que este análisis cobra especial sentidoen un orden regional que parecieratransformarse como resultado de la interconexión entre el cierre de fronteras y el carácter restrictivo de las políticas de control migratorio en el Norte Global, el proceso de regionalización de la política migratoria en Sudamérica y, la aparición de nuevas rutas migratorias y, por lo tanto, nuevos migrantes en diferentes países de la región(Feldman-Bianco, 2015). De este modo, la migración haitiana se sitúa en una coyuntura histórica particular en la que las migraciones internacionales presentan marcadas diferencias con respecto a décadas anteriores, donde los flujos migratorios se orientaban principalmente del Sur al Norte Global. Así, a fines del siglo XX,en el marco de la reestructuración neoliberal, se producen cambios económicos, sociales y políticos relevantes que, intensificados con la crisis de 2008-2009, repercuten en las formas y modalidades que adquieren los movimientos de personas a nivel mundial (Feldman-Bianco, 2018). De esta manera, mientras que el endurecimiento de las políticas de control del Norte Global pareciera incidir en el re direccionamiento de ciertos flujos migratorios,al mismo tiempo,el proceso de regionalizaciónde la política migratoria-bajo el modelo de gobernanza-marca la emergencia de nuevas formas de clasificar y organizar a la migración y a los migrantes $^{2}$ (Domenech, 2015).

En este contexto, se busca articular teóricamente las categorías de fronteras, política de visado, securitización e ilegalización en conexión con el proceso de regionalización de la política migratoria en Sudamérica. En primer lugar,las fronterasson entendidas como históricas, heterogéneas, móviles y flexibles, producidas a partir de la lucha, negociación y conflictoentre múltiples actores, discursos y movimientos, referidano soloa las lógicas gubernamentales sino también las trayectorias de la migración (De Genova, Mezzadra, y Pickles, 2015; Mezzadra y Neilson, 2017) Aquí, en particular, se propone hacer foco en cómo se producenlos procesos de deslocalización delcontrol fronterizo que emergen como resultado del aumento de las prácticas previas al viaje en las que se evalúan a los potenciales migrantes, lejos del territorio real del Estado, en espacios donde la burocratizacióngenera situaciones en las que los derechos de movilidad resultanmás difíciles de invocar (Salter y Multlu, 2010).En este sentido,la 
política de visas es considerada como la forma en que los Estados regulan la entradaa sus territorios y, al mismo tiempo, como una herramienta política especialmente útilen la gestión de las poblaciones móviles (Salter, 2006). Esto en la medida que, al administrar el tipo de visitas (condiciones de trabajo, documentos y/o capital necesario) los Estados establecen fronteras entre porosas, abiertas, cerradas y/o impenetrables. De acuerdo con Salter y Multu (2010), la política de visado es:

un espejo que refleja cómo el Estado ve a su propia población y al mundo: los Estados que perciben su sistema de bienestar como una carga excesiva pueden tener regulaciones estrictas para la admisión de refugiados o mano de obra poco calificada; los Estados que están preocupados por las tasas de fertilidad, por otro lado, pueden fomentar la inmigración; los Estados que dependen del turismo pueden reducir las barreras de visa; los Estados que pueden identificar poblaciones particulares de alto o bajo riesgo pueden optar por facilitar o dirigirse a esos grupos perfilados, con visas de entrada múltiple a largo plazo o restricciones nacionales (Multu, 2010, p.1, traducción propia).

Entendida de esta manera, la política de visado es una de las formas a través de la cual se produce el desplazamiento o desterritorialización del control fronterizo y, en consecuencia, permite observar cómola movilidad de haitianos en la región se conforma como un caso (entre otros) que expresa de manera ejemplar aquella contradicción existente entre el derecho absoluto de salir del país de origen con el derecho restringido para ingresar a los Estados deseados por los migrantes (Salter y Multlu, 2010).

Por su parte, la noción de securitización hace referencia a la vinculación de un fenómeno político y social con la esfera de la seguridad. Es decir, implica la construcción de un fenómeno determinado a partir de su presunta peligrosidad y, por lo tanto, “amenaza” (Campesi, 2012). En esta línea, el análisis de las fronteras a través de la política de visado, se realiza en un marco en el que la migración es construida como un problema político y de seguridad, donde los Estados aceptan "la movilidad de capital y la movilidad de los ricos, de los consumidores en tránsito, de los turistas ricos, pero rechazan la misma libertad para los pobres, las personas que huyen de desastres ecológicos, económicos o políticos” (Bigo, 2004, p.4, traducción propia). Aquí,Sudamérica no representa una excepción al control y,como se verá, la imposición de visados pareciera configurarse comorespuesta estatal inmediata a la llegada de grupos nacionales no “deseables”,dando lugar a una regulación selectiva quenormaliza la criminalización de los migrantes a través de argumentos securitarios y/o humanitarios. De acuerdo con esto, se analizaran diferentes categorías clasificatoriasy prácticas que, alineadas al discurso de las “nuevas amenazas” retratadas por la comunidad internacional (terrorismo, narcotráfico, la trata de personas, el tráfico ilícito, etc,), los Estados reproducen mediante fundamentos securitarios y humanitarios de control (Domenech, 2015). Se considera que las formas en que tales categorías se construyen, condicionan y dialogan con las trayectorias con- 
cretas de los sujetos migrantes permiten avanzar en la comprensión del vínculo entre movilidad, fronteras y seguridad.

\section{La política de visado en Brasil y Chile}

Con relación a la migración haitiana en Brasil, se calcula que, entre febrero de 2010 y julio de 2013, alrededor de 15 mil haitianos ingresaron al territorio (Nieto, 2014) mientras que, para el año 2017, los haitianos ya se posicionaban en el quinto lugar entre los grupos nacionales de mayor presencia luego de Portugal, Bolivia, Japón e Italia. En este país, cuandoel arribo de haitianos cobra impulso a partir del terremoto de Haití en 2010, el ingreso se daba fundamentalmente por vía terrestre (por pasos no autorizados) y, una vez dentro del territorio brasilero, se realizaba la solicitud de refugio.La entrada de haitianos a este país estuvo asociadacon la idea de “invasión” en las fronteras y migración “ilegal” o “clandestina”, conla llegada de enfermedades como el SIDA, el cólera y la malaria, la creación de situaciones de “emergencia” o “crisis humanitaria”y polémicas-políticas y mediáticas- en torno a cuál era el status migratorio que les correspondía tras no encuadrar en las categorías estatales de "migrantes económicos" ni “refugiados” (Nieto, 2014; Silva, 2015; Thomaz, 2013; Véran et al.).

En este escenario, en marzo 2011, se autoriza a través de la Resolución08/06 del Consejo Nacional de Inmigración (CNIg), el otorgamiento de residencias permanentes por razones humanitarias a los haitianos que ya se encontraban en Brasil. Más tarde, en enero de 2012, la Resolución 97/2012 habilita la emisión de visas humanitarias a través del consulado de Brasil en Puerto Príncipe con un cupo de 100 visas por mes, durante cinco años. Posteriormente, en abril de 2013, ante la excesiva demanda y dificultades derivadas de la exigencia de realizar el trámite exclusivamente en Puerto Príncipe, se emite una nueva resolución (112/2013) en la que se eliminan los cupos y se incorpora la posibilidad de tramitar el visado humanitario en Ecuador, Bolivia y República Dominicana (Metzner, 2014). Por su parte, el gobierno de Perú a partir del 2012 comienza a exigir visa de turismo a los ciudadanos haitianos que ingresaban al territorio. Teniendo en cuenta que Perú, previo Ecuador ${ }^{3}$, era una de las principales rutas hacia Brasil, la medida se asocia con la presión ejercida por Brasil al gobierno peruano en el marco de "procesos de cooperación” entre Brasil y los países considerados de tránsito hacia Brasil.

Según Nieto (2014), en 2012, las consecuencias prácticas de estas medidas, más allá del sentido humanitario declarado, fueron:

"el impedimento del ingreso de ciudadanos haitianos sin visa por las fronteras brasileñas, la militarización de la frontera para frenar el paso de haitianos hacia la ciudad de Brasileia y Manaos y la prohibición, a los ciudadanos haitianos, de entrar y transitar, sin visa, por territorio peruano” (p. 61). 
Por lo tanto, durante el 2012, el cierre-apertura de fronteras de Brasil, la existencia de un cupo y la imposibilidad de realizar la solicitud de visa en otro lugar que no fuera Puerto Príncipe se sumó a la imposición de visas de turismo en Perú. Así, el resultado fue el aumento del "ingreso irregular” por pasos no "autorizados", la proliferación de “coyotes" (en Brasil y en Perú) y la creación de situaciones de extrema vulnerabilidad, sobre todo, en las ciudades fronterizas de Perú y Brasil (Nieto, 2014). Más tarde, en 2013, de acuerdo con el análisis de Silva (2015) y Fernandes y Faria (2017), la posibilidad de tramitar visas en Ecuador, Bolivia y República Dominicana, la eliminación del cupo y la apertura de la frontera con Perú no logró resolver la situación debido a la ausencia de infraestructura necesaria para atender los pedidos de visados, la falta información, demoras y costo del mismo, entre las razones principales mencionadas.Así, se sostiene, que la "emergencia humanitaria" en las ciudades fronterizas, lejos de mejorar, se agudizó (Silva, 2015).

Estos análisis permiten delinear las condiciones de emergencia y los efectos prácticos del visado humanitario en Brasil. No obstante, se considera que la cuestión central no remite a la imposibilidad del visado humanitario para resolver la "emergencia humanitaria"en las ciudades fronterizas sino a quela "emergencia humanitaria" o bien, existe precisamente a consecuencia de la implementación del visado o, en todo caso, debe ser analizada a partir de sus relaciones y tensiones con los preceptos securitarios, en tanto que, como se verá más adelante, es por medio de esta categoría, junto a otras, que se construyen las condiciones de posibilidad para la aplicación de prácticas securitarias asociadas al reforzamiento del control en las fronteras. De este modo, lavisa humanitaria, fundada en la supuesta intención de ayudar a los haitianos que querían migrar a Brasil y que se encontraban en una situación de extrema vulnerabilidad debido al terremoto de Haití de 2010 y, de igual modo, la "emergencia humanitaria" con la que se caracterizaba a las ciudades fronterizas, no pueden ser comprendidas sin remitir al plano de la seguridad y al interés por restringir el ingreso de haitianos al país. En este sentido,sin pretensión de igualar la lógica del visado humanitario con otros tipos de visado (como el visado de turismo),interesa señalar que, más allá de las limitaciones propias de la normativa vigente por esos años en Brasil ${ }^{4}$, la denominación "humanitario” no garantiza por sí misma la ausencia de mecanismos estatales restrictivos y selectivos. Así, el visado humanitario, se configuró comouna prácticamás de control migratorio y fronterizo que, con el objetivo de desterritorializar el control hacia los consulados en origensupusola puesta en marcha de dispositivos de "gestión" o regulación delas movilidadesque reafirmanlaposición delEstado como productor de la irregularidad migratoria.

Por su parte, en Chile, en 2013 se calculaba que había alrededor de 5.000 haitianos en el país mientras que,entre 2013 y junio de 2016, se constata un ingreso de 41.065 haitianos (Pedemonte et al. 2015) y, en 2017, se registran más de 100.000 ingresos (Ferrer Durán, 2018). De algún modo,estas cifras señalan elre direccionamiento de losdesplazamientos de haitianos que inicialmente se dirigían hacia Brasil (donde la crisis económica de 2015 
comienza a minar las posibilidades laborales)y, al mismo tiempo, el aumento de la dinámica intrarregional y extra-regional, en tanto comienza a advertirse un mayor movimiento entre los países sudamericanos (sobre todo, hacia Chile) y una movilidad creciente hacia Estados Unidos vía Centroamérica.

A diferencia de lo ocurrido en Brasil, el ingreso de haitianos a Chile,desde el 2010,se produce fundamentalmente por vía aérea, en particular, por el aeropuerto de Santiago de Chile (Pedemonte et al., 2015).Aquí, el aumento progresivo de la llegada de haitianos y su crecientenotoriedad tiene lugar en una sociedad atravesada por marcados procesos de racialización (Tijoux y Córdova, 2015). De acuerdo con Pedemonte et al. (2017), desde el año 2012, comienza a implementarse una exigencia institucional “informal” para los ciudadanos haitianosque consistía en presentar una carta de invitación y 1000 dólares a la hora de ingresar al país (entre otros requisitos que se definían discrecionalmente por los agentes migratorios al momento de ingreso -o, intento de ingreso- por el aeropuerto de Santiago de Chile). Esta práctica tuvo comoresultado el rechazo en frontera del 50\% de las personas de nacionalidad haitiana que quisieron entrar a Chile durante ese año.A pesar de ello, los flujos migratorios de haitianos hacia Chileno se detuvieron sino que, por el contrario, en el 2013 el número de ingresos de este grupo nacional se duplica. Esto no resulta tan extraño si se tiene en cuenta los efectos (recurrentes) que tienen este tipo de políticas restrictivas sobre losmovimientos migratorios reales,en tanto los sujetos no renuncian a sus proyectos migratorios sino que realizan sus experiencias de circulación desde posiciones más vulnerables y/o precarizadas.

En este contexto, en abril de 2018, se lleva adelante el proyecto de reforma migratoria en el cual se propone un tratamiento selectivo y diferencial paralos nacionales haitianos, estableciéndose como medida administrativa de efecto inmediato un "Visado Consular de Turismo Simple” (válido solo por 30 días) y un "Visado Humanitario de Reunificación Familiar", cuyas solicitudes deben ser realizadas exclusivamente en la Embajada de Chile en Haití ${ }^{5}$.Estas medidas de efecto administrativo inmediato perjudicaron gravemente a los haitianos que ya habían sacado sus pasajes hacia Chile y que no podían viajar por no contarcon el visado exigido. Asimismo, el carácter discriminatorio de la medida aparece en la definición misma de los visados. Como señalan en un comunicado de prensa diferentes organizaciones migrantes y pro migrantes de Chile $^{6}$, en primer lugar, un visado humanitario de reunificación familiarno debería ser otorgado por origen nacional sino por situaciones de crisis (razones humanitarias)sin mencionar que, además, se establece de un cupo anual para el mismo. En segundo lugar, el carácter selectivo de la medida se manifiesta al considerar que, a excepción de los dominicanos, a los haitianos es a la única nacionalidad a la que se le imponen requisitos adicionales para solicitar la visa con fines turísticos, aun cuando numéricamente, en aquel momento,se trataba del sexto grupo nacional con mayor presencia en Chile.

Al mismo tiempo, la medidatiene como antecedente una experiencia análoga y reciente de la que ya han sido conocidas sus consecuencias 
prácticas pero sobre la que pareciera no haber consideración. En efecto, en el año 2012, se estableció una exigencia similar para las personas de nacionalidad dominicana cuyo efecto no fue la disminución del flujo migratorio sino su ingreso por pasos no autorizados. A partir de la imposición de la visa consular a ciudadanos de República Dominicanaaumentó un 4550\% (2012 - 2016)el ingreso por pasos no habilitados,fomentando así el negocio de las redes de tráfico (Espinoza, 2018). Por otra parte, si se atiende a las cifras generales de rechazos en frontera ${ }^{7}$ en Chile, se puede observar que "aquellos flujos con presencia importante de población afrodescendiente coinciden con las mayores tasas de rechazo, siendo los haitianos y, en segundo lugar los dominicanos, aquellos que son rechazados con mayor frecuencia en el ingreso" (Pedemonte y Dittborn, 2016, p.15). De este modo, los rechazos de haitianos en Chile son considerados como un preanuncio de su actual política de visado.

\section{La política migratoria argentina y las "nacionalidades sensibles"}

En el caso de Argentina, en 2010, la población haitiana representaba un número marginal con relación a la población total extranjera, siendo registrados solo 309 haitianos al momento de realización del Censo Nacional de Población de dicho año (Del Águila, 2017). Posteriormente, a diferencia de lo ocurrido en otros países como Ecuador y Brasil, en Argentina no se observa un salto cuantitativo inmediato con relación a la llegada de haitianos post-terremoto 2010,sino que su presencia va cobrando mayor visibilidad a partir 2015 y en los años que siguen ${ }^{8}$.Con relación al ingreso, hasta agosto de 2018, los haitianos no requeríansolicitud previa de visadosino que podíaningresar como "residentes transitorios" bajo la subcategoría de turista. De acuerdo conlas entrevistas realizadas a haitianos residentes en este país ${ }^{9}$ y los estudios consultados (Duffard, 2016; Del Águila, 2017; De Heusch, 2016),el arribose realizaba principalmentepor vía aérea, teniendo como punto de partida Haití o República Dominicana. En este último caso,el trayecto Haití-República Dominicanase realizaba por vía terrestre, previa solicitud de visa.

La movilidad hacia Chile y Argentinapor vía aérea, posiciona a los aeropuertoscomo "zonas de frontera"sumamente representativas en las trayectorias de migrantes haitianos en la región.De este modo, los aeropuertos,son entendidos como zonas de filtrado,"zonas sin derecho", donde las garantías de la libertad individual están supeditadas, por más o menos tiempo, y en las que los extranjeros vuelven a ser no ciudadanos y parias:clara ilustración de la paradoja (...) de las condiciones no democráticas de la democracia” (Balibar, 2005, p.94).

Precisamente, los rechazos en frontera en Chile y Argentinaremiten a prácticas estatales que dan cuenta del carácter selectivo que opera en los cruces de frontera y, al mismo tiempo, de la conformación de los aeropuer- 
tos como “zonas de retención y filtro”. Enel caso de Argentina, desde mediados del 2000, el ingreso se venía realizandovía aérea sin grandes sobresaltos, hasta que,en 2017 y, sobre todo, a inicios de 2018, los haitianos comienzan aexperimentarun progresivo aumentode los rechazos en frontera bajo la categoría de "falsoturista”,que cobra mayor notoriedada partir de los meses de abril y mayo del mismo año ${ }^{10}$.En Argentina, la Disposición $N^{0}$ 4362/2014,establecida en el año 2014, fija un "Procedimiento para la Resolución de casos sobre sospecha fundada en la Subcategoría Turista”, en el que se pautan una serie de criterios de $\operatorname{control}^{11}$ y pedido de documentación que pueden ser complementados con aquello que el agente migratorio considere necesario para probar la correspondencia del migrante con la figura de turista (Defensoría del Pueblo, 2016).

Aquí, al igual que en Chile, los rechazos en frontera ${ }^{12}$ serán un anticipo de la actual política de visado. Los datos oficiales sobre este tema son escasos y dispersos pero a raíz de la imposición del visado de turismo, diversas fuentes periodísticas publicaron cifras y declaraciones de funcionarios de la DNM. Esto, en sintonía con lo que ya aparecía explicitado en la Resolución477/2018 delMinisterio del Interior, Obras Públicas y Vivienda,que reglamenta dicho visado y donde se señalan dos motivos fundamentales para la implementación del mismo: el aumento de los rechazos en frontera por “falso turista”y el aumento de las solitudes de refugio sin resoluciones favorables.

Sobre el primer punto, en una de estas notas periodísticas, funcionarios de la DNM explicaban que la medida había sido tomada para "regular el ingreso” de inmigrantes haitianos ante el aumento del número que llegaba al país sin encuadrar en la categoría de turista. Estoera sintetizado por el medio periodístico de la siguiente manera:

Los números son elocuentes: según datos de la Dirección Nacional de Migraciones, 71 haitianos fueron rechazados en frontera durante 2016. En 2017, la cantidad subió a 221.Este año, las inadmisiones se multiplicaron: solo entre enero y julio, 900 ciudadanos de Haití tuvieron que volverse a su país por ser considerados 'falsos turistas' en aeropuertos argentinos. La previsión para 2018 era de un aumento del 400\% en rechazos respecto al período previo (Lozano, 2018).

Aquí, el discurso estatal y mediático sostiene como primer argumento para la imposición del visado de turismo el aumento de los rechazos en frontera bajo la categoría del "falso turista", en el marco de una narrativa en la que los propios migrantes serían los culpables por no cumplir con los requerimientos de la figura de turista y, en ese sentido, estarían falseando la información sobre sus verdaderos fines para arribar al país. Ahora bien, esta narrativa pasa por alto varios puntos, por ejemplo,que la Ley Nacional de Migraciones 25.871 permite el cambio de status migratorio, es decir, se puede ingresar como turista y luego tramitar la residencia temporaria por otras razones o, también, que los cruces de frontera se realizaban muchas veces sin contar con intérpretes necesarios para realizar las preguntas exi- 
gidas por "test de turista". Pero, además, evita mencionar el trasfondo del porqué 900 ciudadanos haitianos fueron rechazados entre enero y julio del 2018 y porqué la previsión para el 2018 esun aumento del 400\% de rechazos en frontera por "falso turista". Este trasfondo tiene que ver con una decisión institucional que habilita, legitima e impulsael accionar discrecional de los agentes migratorios de distintos aeropuertos de Argentina. En efecto, el 10 mayo de 2018,la DNM emitióel Memorándum N 192/18, en el que se exigía la aplicación de las figura del "falso turista" a haitianos y "otras nacionalidades sensibles”. La finalidad de este memorándum se explicitaba de la siguiente forma:

Asunto: Sospecha fundada en la subcategoría turista (haitianos y otras nacionalidades sensibles).Por medio de la presente se reitera a todo el personal la obligación de extremar los recaudos ante el ingreso de nacionales haitianos y/o cualquier otra nacionalidad sensible (de medio oriente, colombianos, africanos, etc.) a la República Argentina. Que la disposición DNM N 4362/14 acerca del Procedimiento ante Sospecha Fundada en la Subcategoría Turista, establece un protocolo de evaluación de pasajeros, por lo cual la falta de examinaciónola examinación deficiente serán consideradas faltas graves (Memorándum Nº 192,2018.Énfasis del texto original).

De esta manera, el aumento de rechazos en frontera bajo la categoría de “falso turista”fue el desenlacelógico de la directriz impartida por la DNM a través del Memorándum, una situación creada por los propios mecanismos estatales de regulación que responde más al aumento de los ingresos de haitianos al país (y, a su intento de restricción) que a las supuestas discordancias con el encuadre de turista, como puede constatarse en los numerosos testimonios de entrevistados haitianos cuyos familiares, amigos y conocidos fueron rechazados a pesar de cumplir con los requerimientos de "turista". Asimismo, se considera que la definición de "nacionalidades sensibles" entre las que se incluyen las de ciertos países (Haití y Colombia), pasando por regiones (como Medio Oriente), hasta un continente en su totalidad (África), refleja de manera manifiesta los criterios institucionales que operan en la regulación diferencial de la movilidad a través de las fronteras.

En segundo lugar, con relación al aumento de las solicitudes de refugio, la Resolución 477/2018puntualiza:

Que, por otro lado, la COMISIÓN NACIONAL PARA LOS REFUGIADOS (CONARE), organismo actuante en la órbita de este Ministerio, ha informado que desde mediados del año 2017 se ha observado un incremento notable de las solicitudes de refugio de ciudadanos haitianos, habiendo tenido DOSCIENTAS NOVENTA Y CUATRO (294) solicitudes en el año 2016, TRESCIENTAS OCHENTA Y CINCO (385) en el año 2017 y totalizando TRESCIENTAS TREINTA Y OCHO (338) solicitudes en lo que va del año 2018(Resolución $N^{\circ}$ 477, 2018.Énfasis del texto original). 
Aquí, nuevamente cobra relevancia no tanto elaumento de las solicitudes de refugio sino el porqué de este aumento (y denegación de la mayoría de ellas), una situación que también es producto del funcionamiento de mecanismos estatales de control, en este caso, que obstaculizan las posibilidades de radicación en Argentina de aquellos migrantes clasificados como “Extra-Mercosur”.Como analizan Nejamkis y Nievas (2012), la correlación entre la política migratoria y la política de asilo y refugio puede ser comprendida, en parte, a partir de las dificultades que tienen ciertos grupos nacionales de acceder a la regularidad migratoria, utilizando las solicitudes de refugio como estrategia para permanecer en el país de manera "regular". En este sentido, se genera“"un limbo jurídico para aquellos inmigrantes que carecen de criterio migratorio o de los requisitos para obtener la condición de refugiado" (p.447). Sin entrar en mayor detalle sobre este tema, bastacon remarcar nuevamente el papel del Estado en la producción de situaciones que, luego, son utilizadas como argumentos para la implementaciónde diversas prácticas de control, en este caso, de una política de visado orientada a restringir el ingreso de ciudadanos haitianos a la Argentina.

\section{La frontera como mecanismo de selección}

Más allá de las particularidades de los marcos nacionales y sus respectivas normativas, lo cierto es que la migración haitiana tiene lugar en Sudamérica en el marco de un proceso de reconfiguración política del mapa migratorio regional, en el que, desde mediados del 2000, se advierten variadas prácticas de control en torno a las nuevas movilidades de personas. Como se mencionaba al inicio, junto a la aparición de "nuevos grupos migratorios" emergen categorías clasificatorias que ponen en marcha complejos mecanismos de inclusión/exclusión que, de acuerdo a los diferentes contextos históricamente situados, permiten abrir nuevos interrogantes entorno al nexo entre movilidad, fronteras y seguridad. En este sentido, la llegada de migrantes provenientes de África, América central y El Caribe entre otros-aparece ligada a la producción de diversas categorías que, alineadas al discurso de las "nuevas amenazas" retratadas por la comunidad internacional, los Estados reproducen y refuerzan a través de fundamentos securitarios y/o humanitarios de control ${ }^{13}$ (Domenech, 2015).

En palabras de Campesi (2012), "la securitización es el proceso mediante el cual una cuestión viene transformada en un problema de seguridad, con total independencia de su naturaleza objetiva, o de la relevancia específica de la supuesta naturaleza” (p.5).Como ha sido analizado, en el espacio sudamericano, la gobernabilidad migratoriano se ha estructurado como alternativa al enfoque securitariosino más biendesde sucomplementariedad e imbricación con el paradigma humanitarista (Domenech, 2013). En esta línea, la noción de "políticas de control con rostro humano" hace referencia

al universo de ideas y prácticas que, enmarcadas en el discurso de los derechos humanos como fuente de legitimación, persiguen la 
misma finalidad que las políticas más abiertamente restrictivas, prometiendo ser más eficaces en la consecución de su objetivo: controlar los flujos migratorios internacionales. (Domenech, 2013, p.121).

En este escenarioaparecela construcción de la "migración haitiana"como potencial amenaza asociada a su irregularidad migratoria.Esto, ya sea por suingreso de manera "ilegal" por pasos no habilitados o por su ingreso como turistas que finalmente se quedan en el país "abusando del sistema” (de salud, de educación, habitacional, etc.). Por otro lado, las prácticas de vigilancia y control sobre este grupo nacional son legitimadas por discursos basados en la defensa de los derechos humanos y la lucha contra la trata de personas y el tráfico ilegal de personas. En esta segunda línea de argumentación, el migrante es construido (de manera simultánea) como víctima.

En el caso de Brasil, la política de visado humanitario se presentaba como orientada a la protección de los derechos humanos de los migrantes, por medio de la apertura de un canal formal y regular y, al mismo tiempo, con relación al efecto que estas medidas tendrían en la lucha contra las redes de trata y tráfico ilegal de migranteshaitianos.De acuerdo con algunas declaracionesrealizadas por funcionarioscon relación a los visados humanitarios en diferentes medios periodísticos, se sostenía por ejemplo:

Brasil no tiene esa postura (de cerrar las fronteras), ni la de hacer deportaciones masivas. No cerramos nada, sólo estamos regularizando la entrada de esas personas, para evitar que corran riesgos con las redes internacionales de tráfico humano" ("Brasil regulamenta concessão de vistos para imigrantes haitianos”, 2012. Traducción propia.)

Más tarde, en 2014 y 2015:

Un haitiano que viene con visa, puede llegar en avión a Brasil sin usar un coyote, sin estar sujeto a la explotación, y por tanto, sin estar sujeto a un drama humano, sin tener que atravesar varios países hasta entrar, y crear dificultades logísticas (Brasil acelerará visas para haitianos y evitar que sean víctimas de “coyotes”, 2014)

Nuestro gran desafío, hoy, es transformar una ruta muy indeseada de sumisión de inmigrantes a 'coyotes' ya organizaciones criminales, y, por lo tanto, someterlos a posibles violaciones de derechos humanos - a una ruta que sea segura, que garantice el trato humanitario, acogida e inserción social, laboral y cultural de esos inmigrantes a Brasil (Marcel, 2015. Traducción propia.).

Teniendo en cuenta el análisis de las implicancias que tuvo el visado humanitario para gran parte de los haitianos que se dirigían a Brasil y, a pesar de serconsiderado un antecedente fundamental para el posterior tratamiento de la nueva ley migratoria de Brasil de 2017 (Fernandes y Faria, 
2017),se considera que, las supuestas “deficiencias” del visado (como pueden ser aquellas referidas a su complejidad burocrática y la falta de infraestructura para atender los pedidos),no remiten a un problema “externo" del funcionamiento del mismo sino que, por el contrario, forman parte de la lógica misma de desterritorialización del control fronterizo. Como ya se mencionó, el visado humanitario supusola puesta en práctica de lógicas punitivas de control que, como muestran las diferentes declaraciones citadas,encontraron su fuente de legitimación en los discursos y prácticas humanitarias, es decir,en la preocupación por la "trata y tráfico ilegal de personas”,las “organizaciones criminales”y, en general, en la necesidad de garantizar el cumplimiento de los derechos humanos de los migrantes haitianos.De este modo, se sostiene que, las relaciones entre humanitarismo y securitización,no necesariamente son de oposición sino que se encuentran ligadas a múltiples tensiones, espacios y actores (Domenech, 2018). En esta línea, Fassin (2005) refiere auna “economía moral” dela política migratoria que oscila entre sentimientos de simpatía de un lado y preocupación por el orden por otro, entre una política de la lástima y una política del control que, muchas veces, invoca la idea de “crisis” o “emergencia humanitaria” para justificar medidas “draconianas” de protección de las fronteras.

Por otra parte, los casos de Chile y Argentina no dejan dudas sobre el carácter exclusivamente restrictivodel visado de turismo para ciudadanos haitianos. Sin embargo, en ambos paísestambién se intenta legitimar la medida mediante los mismos fundamentos. En el caso de Chile, por ejemplo,el Ministro del Interior, que respondía a las críticas sobre el carácter discriminatorio de la medida, se expresaba en un medio de la siguiente manera:

Creo que es una equivocación el pensar que aquí hay un trato discriminatorio, sino que por el contrario, lo que se está haciendo precisamente es por una parte, buscar proteger a todas las personas que vengan desde Haití para que no sean traídas bajo engaño, bajo estafa, a veces con posibilidades hasta que puede haber tráfico de personas, y así para que en el ámbito laboral puedan venir en condiciones que sean realmente acorde a su dignidad como personas(Marín, 2018).

A continuación, recalcaba que la medida se había pensado no solo para resguardar la seguridad de los extranjeros de ese país, sino también en defensa de la seguridad nacional:

(Debemos) proteger a nuestro país de que no lleguen personas que dicen que vienen como turistas, sabiendo que no vienen como turistas y sin que nosotros tengamos tampoco la información de sus propios antecedentes o las situaciones personales en el ámbito penal que les pueden afectar. (Marín, 2018).

Asimismo, estas declaraciones referidas al caso haitiano en particular, responden a la orientación general de la reforma del proyecto de ley en Chile que, bajo el fundamento de una "migración segura, ordenada y regu- 
lar”, argumenta su carácter de urgencia en base a las supuestas consecuencias negativasque tendría para el país la entrada de aquellos que ingresan como turistas y se quedan de manera "irregular”, entre ellas, “precariedad laboral y habitacional, incremento de la trata de personas y abuso del sistema, ya que las personas que ingresan mienten sobre el objetivo de su visita al país” (Minuta Reforma Migratoria de Chile, abril de 2018).

Por su parte, en el caso de Argentina, la resoluciónque reglamenta el visado de turismo para haitianos argumenta su necesidad "en el marco de las políticas que viene implementando el PODER EJECUTIVO NACIONAL destinadas a regular el ingreso de inmigrantes al país, buscando garantizar un tránsito seguro, ordenado, eficiente y ágil” (Resolución N 477, 2018. Énfasis del texto original).

De este modo, en la misma línea de “ordenar los flujos migratorios” se suman a las causas mencionadas en el apartado anterior (sobre el aumento de rechazos por "falso turista" y aumento de las solicitudes de refugio),otros argumentosrelacionados con la trata de personas y las posibles actividades delictivas en las que podrían incurrir los migrantes por su condición “irregular”. Estos fundamentos aparecen en las declaraciones de funcionarios de la DNM en distintos medios periodísticos para justificar la medida del visado. En uno de ellos, por ejemplo, se expresaba:

No es una restricción, sino una medida para evitar que las personas que evidentemente no están en condiciones de realizar las actividades que dicen venir a realizar se encuentren con el inconveniente de que las rechacen en la ventanilla de migraciones (Lozano, 2018).

\section{Y, a continuación:}

El objetivo es también proteger a estas personas que llegan en situación de vulnerabilidad, quienes muchas veces son utilizadas por tratantes y proxenetas. Otros pueden terminar realizando actividades delictivas, como la venta ilegal. (Lozano, 2018).

De esta manera, tanto en Chile como en Argentina, en torno a la misma medida se conjugaban argumentos, por una lado, humanitarios, referidos al tráfico ilegal y la trata de personas, ya que, mediante el combate “irregularidad migratoria”,el visado serviría para proteger simultáneamente la seguridad del Estado y la de los migrantes. Y, por otro lado,con argumentos de "seguridad",en referencia a supuesto abuso del sistema por parte de haitianos que ingresaban como turistas y luego se quedaban en situación “irregular” suponiendo un riesgo también parael Estado. En cualquier caso,como puede advertirse, el desenlace de estos discursos resulta en la necesidad de “ordenar" los flujos migratorios mediante el reforzamiento de las prácticas de control y vigilancia sobre este grupo nacional.De tal manera, las estrategias de securitización aparecen tanto en términos de protección, el migrante como "víctima”, como en términos de criminalización, el migrante como “amenaza”(Magliano y Clavijo, 2013). 
Así, como analiza Pereira (2018), algunas formas de humanitarismo conviven con la construcción de las migraciones como riesgo para la seguridad y, en este sentido, "políticas o discursos que en principio tienen como finalidad la protección de un grupo de individuos pueden producir efectos totalmente diferentes a los de protección o defensa de los derechos humanos”(p.3).Tal como afirmaMansur Dias (2017),

aunque se presenta bajo el ropaje de los derechos humanos y la protección de las víctimas, los protocolos de tráfico ilícito de migrantes y la trata de personas reiteran el vínculo entre la migración y la seguridad, reforzando prácticas de criminalización y el control migratorio (p.542).

Teniendo en cuenta estos elementos, pareciera advertirse que la construcción socio-política de la figura del migrante haitiano como víctima y, en términos más generales, de los haitianos como grupo social vulnerable, estaría promoviendo la aplicación medidas estatales que, en concreto, demandan un mayor control fronterizo sobre este grupo nacional específico, convirtiendo así la "frontera" en un mecanismo fundamental de selección y a la política de visado en una forma dedeslocalizacióndel control fronterizo que sitúa al mismofuera del territorio real del Estado, donde la burocratización de la toma de decisiones resulta en una gran dificultad para hacer valer los derechos de movilidad (Salter y Maltlu, 2010). Por tanto, el uso de fundamentos humanitarios -como la trata y el tráfico ilegal- al traducirse también en la aplicación deestrategias de mayor control,estaría contribuyendo al reforzamiento del vínculo entre migración y seguridad sin ninguna base empírica que demuestre de qué manera el aumento del control fronterizo (a través de visados con requisitos imposibles de cumplir y rechazos en frontera que libran a su suerte a los migrantes) estaría contribuyendo a disminuir la trata o tráfico ilícito de personas. De esta manera, se considera que las trayectorias de los migrantes haitianos hacia y en Argentina y, en general hacia Sudamérica,deben ser abordadas prestando especial atención al tratamiento político que reciben ciertos tópicos en la fundamentación de las prácticas estatales de control y sus implicancias en términos de criminalización e ilegalización sobre grupos nacionales específicos.

\section{Reflexiones finales}

La política de visado se presenta en el espacio sudamericano como una práctica de control migratorio y fronterizo sobre la que aún es necesario indagar en profundidad, de manera sistemática y desde un marco de comprensión histórico, situado y multiescalar. A pesar de ello, se considera que esta aproximación permite observar cómo la aparición de nuevosmovimientos migratorios en la región abre camino a la puesta en marcha de mecanismos selectivos que, bajo complejas formas de inclusión-exclusión, se orientan a restringir la llegada de ciertos grupos nacionales a través de diferentes prácticas estatales de control. En este sentido, resulta necesario advertir cómo los visados, conjugados con prácticas de expulsión (como los recha- 
zos en frontera) y los diversos obstáculos existentes para la obtención de la regularidad migratoria, colocan a los Estados como actores fundamentales, aunque no únicos, de los procesos de ilegalización y criminalización de aquellos migrantes considerados no "deseables". Todo ello, desde una narrativa que afirma contradictoriamente la necesidad de seguridad y humanitarismo pero que, en términos prácticos, resulta, sobre todo, en la vulneración de sus derechos de movilidad. Es decir, priorizando la "seguridad nacional” por encima de la protección de los migrantes.

En este marco, la movilidad reciente de haitianos hacia Sudamérica y su dinámica intrarregional y extra-regional implicó la puesta en marcha de prácticas de control de carácter restrictivo sobre este grupo nacional. De este modo, la consideración de la política de visado como mecanismo fundamental de regulación de las fronteras, referido a las trayectorias migrantes de haitianos en la región, arroja luz sobre algunas de las características que va asumiendo el proceso de regionalización de la política migratoria en los últimos años y las implicancias del modelo de "gobernanza" sobre determinados grupos de población. Así, se propuso poner en evidencia cómo diferentes países de la región convergen en prácticas estatales de control que dan cuenta de la manera en que ciertos grupos nacionales se incorporan a la agenda de seguridad en el espacio sudamericano, bajo el común denominador demigración "segura, ordenada y regular", presente en todos los discursos y prácticas mencionadas. 


\section{Notas}

${ }^{1}$ Entre ellos: Nieto, 2014; Metzner, 2014; Silva, 2015; Fernandes y Faria, 2017; Burey, 2018; Pedemonte, Amode y Rencoret, 2015; Pedemonte, Amode y Vásquez, 2017; Pedemonte yDittborn, 2016;Duffard, 2016; Del Águila, 2017; De Heusch, 2016;Cárdenas, 2015; Alarcón, 2015;Véran, Da Silva yFainstat, 2014; Berganza, 2017; Thomaz, 2013.

${ }^{2}$ Dicha regionalización forma parte de "un proceso global de transnacionalización de la política de migraciones internacionales, impulsado por agencias multilaterales como la Organización Internacional para las Migraciones (OIM) y diversas instituciones de Naciones Unidas, cuyo resultado más notorio es la conformación de un régimen global de control migratorio” (Domenech, 2013, p. 120).

${ }^{3}$ Otra medida que influyó decisivamente sobre las trayectorias de migrantes de haitianos en la región fue la eliminación de las visas para el ingreso a Ecuador en 2008. Mientras que, en 2010, Ecuador contaba con 495 personas de nacionalidad haitiana, entre 2004 y 2013 se registró un ingreso de 23.483 haitianos y un egreso de 7.233 haitianos. Del total de estos ingresos, el 95\% se concentra entre 2010 y 2013 (Alarcón, 2015). Aquí, cabe señalar que frente al aumento de la llegada de haitianos a este país también se observa, como en otros países sudamericanos, un aumento de las prácticas de rechazo en frontera en aeropuertos (Cárdenas, 2015) y medidas “especiales” como la exigencia de tramitar una solicitud de permiso sujeta a evaluación a través de un "Sistema Virtual de Registro Turístico” para ciudadanos haitianos, como anunció el gobierno ecuatoriano en agosto de 2015 a través del Memorando Nro. MREMH-VMH-2015-1980 (http:/ /www.consuladovirtual.gob.ec/web/guest/inicio).

${ }^{4}$ La política de visado actual en Brasil debería analizarse a la luz de la nueva Ley de Migraciones $\left(\mathrm{N}^{\circ} 13.445\right)$ sancionada en mayo de 2017 y las negociaciones posteriores para su reglamentación.

5 Recuperado de https://cdn.digital.gob.cl/filer_public/b0/09/...2ac5.../ nueva_ley_de_migracion.pdf

${ }^{6}$ Recuperado de http://www.revistasur.cl/revistasur.cl/2018/04/comunicado-de-organizaciones-migrantes-y-promigrantes-respecto-al-proyecto-de-ley-de-migraciones/

${ }^{7}$ Los rechazos de haitianos entre 2011 y 2015 van desde un 14,35\% de rechazos sobre el total de ingresos, hasta un 17,46\%, a excepción de 2012 momento en que se registra un pico que llega al 53,34\% de rechazos en frontera de haitianos(Pedemonte y Dittborn, 2016).

${ }^{8}$ Según los datos de la Dirección Nacional de Migraciones (DNM), los trámites de residencias temporarias en los años posteriores al terremoto van desde 135, en 2010, a un máximo de 206 en 2012, volviendo a disminuir al número de 162 residencias temporales en 2013.Mientras que, de acuerdo con el informe realizado por Del Águila (2017), los ingresos al territorio entre 2010 y 2013 se mantienen entre un mínimo de 1004 ingresos y un máximo de 1742, notándose un incremento de 3456 ingresos a partir de 2015, sin contar con datos oficiales sobre los años que siguen. Asimismo, con respecto al número de solicitudes de refugio realizadas, entre 2007 y 2014 el número 
oscila entre un mínimo 11 y un máximo de 63 solicitudes, con un leve aumento en 2015 que alcanza las 109 solicitudes.

${ }^{9}$ Las referencias realizadas sobre entrevistas a migrantes haitianos residentes en Argentina en este documento, remiten a mi tesis doctoral en curso y fueron realizadas entre 2017 y 2018 en las ciudades de Córdoba y Rosario a un total de 30 personas de nacionalidad haitiana.

${ }^{10}$ Diferentes aspectos referidos a la figura del "falso turista" en Argentina han sido abordados con mayor detalle en García (2013), Domenech (2018) y AlvitesBaiadeira (2018).

${ }^{11}$ Entre los criterios explicitados en la denominada "bolsa de viaje" se evalúa la información sobre el lugar de residencia permanente. su actividad en el país de origen, el tiempo que planea quedarse en el país, los puntos turísticos a visitar y las formas de traslado hacia los mismos, de los parientes o personas de su conocimiento y/o amistad que posean residencia en el país. Para ello, “el inspector de control migratorio podrá solicitar al extranjero la presentación de documentación respaldatoria tal como tickets aéreos, tarjetas de crédito, pasajes locales o internacionales por cualquier medio de transporte, reservas de hoteles, y todo otro elemento con igual alcance que el enunciado” (Defensoría del Pueblo, 2016).

12 En charlas informales con dos agentes migratorios de uno de los aeropuertos de Argentina, se justificaba el aumento de los rechazos en frontera por "falso turista" con relación al problema de la "trata de personas", "tránsito ilegal” y "mafias” asociadas al ingreso de haitianos. Categorías vinculadas, además, a las trayectorias de migrantes haitianos hacia Chile y Brasil junto con el señalamiento de que Argentina se había vuelto un país de tránsito para el ingreso "ilegal" de haitianos hacia estos países. De esta manera, el uso de estas categorías si bien en inicialmente no aparecieron como resultado de declaraciones oficiales (en parte, porque los rechazos permanecen como una práctica “oculta” que no cobra visibilidad pública) más tarde, con la imposición del visado de turismo, se trasformaron en los argumentos centrales de esta política selectiva.

${ }^{13}$ En este sentido, cabe señalar que, a escala regional, los grupos nacionales que presentan las estadísticas más elevadas con relación a las expulsiones, deportaciones y rechazos en frontera son los colombianos, chinos, cubanos, haitianos y dominicanos (Domenech, 2015). Datos que habría que analizar, también, a luz de movilidad actual de venezolanos en la región. 


\section{Bibliografía}

Alarcón, M. (2015). Las asociaciones de migrantes haitianos en el Ecuador: entre debilidad y resistencia. REMHU.Revista Interdisciplinar da Mobilidade Humana, 23(44), 207-220.Recuperado de http:// www.redalyc.org/articulo.oa?id=407042021013

Alvites Baiadera, A. (en prensa). Extranjeros bajo la lupa: La figura del "falso turista" en Argentina.Horizontes Decoloniales.

Balibar, E. (2005). Fronteras del mundo, fronteras de la política. Alteridades, 15(30), 87-96. Recuperado de http://www.scielo.org.mx/ scielo.php?pid=S0188-70172005000200087\&script=sci_arttext

Berganza, I. (2017). Los flujos migratorios mixtos en tránsito por Perú: Un desafío para el Estado. En N.Pedemonte y J. Koechlin (eds.), Migración haitiana hacia el sur andino (pp.41-64). Santiago, Chile, Chile: OBIMID.

Bigo, D. (2004). Criminalisation of “migrants": Thesideeffect of thewill to control thefrontiers and thesovereignillusion. n/a. Recuperado de https://s3.amazonaws.com/academia.edu.documents/31303874/ Criminalisation_of_migrants

Burey, R. (2018). Estrategias migratorias en el tránsito de emigrantes haitianos hacia Estados Unidos. Huellas de la Migración, 2(4), 93123. Recuperado de https://huellasdelamigracion.uaemex.mx/article/ view/4604/8005

Campesi, G. (2012). Migraciones, seguridad y confines en la teoría social contemporánea. Revista Crítica Penal y Poder, (3), 166-186. Recuperado de http://revistes.ub.edu/index.php/CriticaPenalPoder/article/ view/3657

Cárdenas, I. (2015). Migraciones haitianas en la región andina (Dossier central).Andina Migrante, (19), 2-13. Quito, Ecuador: FLACSO. Recuperado de http://repositorio.flacsoandes.edu.ec/bitstream/10469/ 6772/1/BFLACSO-AM19-01-Ceja.pdf

De Genova, N.,Mezzadra, S. yPickes, J. (2015). New Keywords: Migration and Borders. Cultural Studies,29(1), 55-87. doi:10.1080/ 09502386.2014.891630

De Heusch, F. (2016). “Amo a mi país, sigo hablando en creole, pero tomo el fernet con coca y como el asado": Una etnografía de los migrantes haitianos y de la venta de "bijouteria" en la ciudad de Córdoba, Argentina (Tesis de Licenciatura). Universidad Nacional de Córdoba, Córdoba, Argentina. 
Defensoría del Pueblo (2016). Informe Rechazos en frontera. La aplicación de la categoría pseudo-turistaen ciudadanos de países del Mercosur. Recuperado de http://www.defensoria.org.ar/wp-content/uploads/ 2016/12/Rechazos-en-frontera-Informe-octubre-2016-FINAL1.pdf

Del Águila, Á. (2017). Estudio exploratorio sobre las trayectorias socioeducativas y socio-laborales de migrantes haitianos, senegaleses y ucranianos en la ciudad de Buenos Aires. (Informe del proyecto "Promoción de la inclusión social de las personas migrantes en Argentina”). Buenos Aires: OIM/FCCAM.

Domenech, E. (2013). ‘Las migraciones son como el agua’: Hacia la instauración de políticas de 'control con rostro humano'. La gobernabilidad migratoria en la Argentina. Polis.Revista Latinoamericana,12(35), 121.Recuperado de https://journals.openedition.org/polis/9280

(2015). Controle da imigração ‘indesejável’: expulsão e expulsabilidade na América do Sul. Ciência e Cultura, 67(2), 2529.doi: 10.21800/2317-66602015000200010

(2017). Las políticas de migración en Sudamérica: elementos para el análisis crítico del control migratorio y fronterizo.TerceiroMilênio.Revista Crítica de Sociologia e Política,8(1), 19-48. Recuperado de https://ri.conicet.gov.ar/handle/11336/ 58156

Domenech, E. y Boito, E. (en prensa). Luchas migrantes en Sudamérica: reflexiones críticas desde la mirada de la autonomía de las migraciones. En B. Cordero, S. Mezzadra y A. Varela (comp.), América latina en movimiento. Migraciones, límites a la movilidad y sus desbordamientos.

Duffard, I. (2016). Del Caribe haitiano a la Argentina: Trayectorias de cuerpos en movilidad humana pos terremoto 2010. Recuperado de http://biblioteca.clacso.edu.ar/clacso/pobreza/20160307040904/ Duffard.pdf

Düvell, F. (2003). Theglobalisation of migration control. Opendemocracy. Recuperado de https://www.opendemocracy.net/peoplemigrationeurope/article_1274.jsp

Fassin, D. (2005). Compassion and repression: the moral economy of immigrationpolicies in France. Cultural anthropology, 20(3), 362387.doi: 101525/can.2005.20.3.362

Feldman-Bianco, B. (2015).Apresentação: deslocamentos, desigualdades e violência do Estado. Ciência e Cultura, 67(2), 20-24.doi: 10.21800/ 2317-66602015000200009 
(2018). Anthropology and ethnography: the transnational perspective o migration and beyond.Etnográfica, 22(1), 195-215. doi:10.4000/etnografica.5203

Fernandes, D. y Faria, A. (2017). O visto humanitário como respostaao pedido de refúgio dos haitianos. Revista Brasileira de Estudos de População, 34(1), 145-161. Recuperado de http://www.scielo.br/ scielo.php?pid=S010230982017000100145\&script=sci_abstract\&tlng=es

García, L. (2013). Nueva política migratoria argentina y derechos de la movilidad. Implementación y desafíos de una política basada en derechos humanos a través de las acciones ante el Poder Judicial (20042010) (Tesis de doctorado). Universidad de Buenos Aires, Buenos Aires, Argentina.

Magliano, M. y Clavijo, J. (2013). La OIM como ‘traffickingsolver’ para la región sudamericana: sentidos de las nuevas estrategias de control migratorio. EnG. Karasik (ed.),Migraciones internacionales contemporáneas. Reflexiones y estudios sobre la movilidad territorial de la población (pp.129-148). Buenos Aires, Argentina: CICCUS.

MansurDías, G. (2017). Trata de personas, tráfico de migrantes y la gobernabilidad de la migración a través del crimen. Etnográfica, 21 (3),541-554. doi: 10.4000/etnografica.5026

Metzner, T. (2014). La migración haitiana hacia Brasil: estudio en el país de origen.La migración haitiana hacia Brasil.Cuadernos migratorios (6). Buenos Aires, Argentina: OIM.

Mezzadra, S. y Neilson, B. (2017). La frontera como método. Madrid, España: Traficantes de sueños.

Nejamkis, L. y Nievas, J. (2012). Migración y refugio en la Argentina: un análisis desde la legislación actual. Passagens, 4(3), 445-463. Recuperado de https://dialnet.unirioja.es/servlet/articulo?codigo $=4017483$

Nieto, C. (2014). Migración haitiana a Brasil: Redes migratorias y espacio social transnacional. Recuperado de http://biblioteca.clacso.edu.ar/ clacso/se/20141118015558/Migracion.pdf

Pedemonte, N., Amode, N. y Rencoret, J. (2015). Racismo y matrices de “inclusión” de la migración haitiana en Chile: elementos conceptuales y contextuales para la discusión. Polis.Revista Latinoamericana,14 (42), 217-245. Recuperado de https://journals.openedition.org/polis/11341

Pedemonte, N. Amode, N. y Vásquez, J. (2017).Migración haitiana hacia Chile: origen y aterrizaje de nuevos proyectos migratorios. En N.Pedemonte, y J. Koechlin (eds.), Migración haitiana hacia el sur andino (pp.65-172). Santiago de Chile, Chile: OBIMID. 
Pedemonte, N. y Dittborn, C. (2016). La migración en Chile: breve reporte y caracterización. (Informe del Observatorio Iberoamericano sobre Movilidad Humana, Migraciones y Desarrollo). Madrid, España: OBIMID

Pereira (en prensa). La relación entre migración, seguridad y derechos humanos en la política migratoria Argentina 1990-2015: humanitarismo de expiación, humanitarismo de contestación y humanitarismo de prevención. Desafíos.

Salter, M. B. (2006). The global visa regime and the political technologies of the international self: Borders, bodies, biopolitics. Alternatives, 31(2), 167-189. doi: 10.1177/030437540603100203

Salter, M. y Mutlu, C. E. (2010). Asymmetricborders: TheCanadaCzechRepublic 'visa war' and thequestion of rights. CEPS.Liberty and Security in Europe. Recuperado de http://aei.pitt.edu/15127/1/ Salter_\&_Mutlu_on_Asymmetric_Borders.pdf

Silva, S. (2015). Fronteiraamazónica: passagemobrigatória para haitianos? REMHU.Revista Interdisciplinar da Mobilidade Humana, 23(44), 119-134. Recuperado de http://www.scielo.br/ scielo.php?pid=S198085852015000100119\&script=sci_abstract\&tlng=pt

Tijoux M. E y Córdova, M.G. (2015). Racismo en Chile: colonialismo, nacionalismo, capitalismo. PolisRevista Latinoamericana, 14(42), 713. Recuperado de https://scielo.conicyt.cl/ scielo.php?script=sci_arttext\&pid=S0718-65682015000300001

Thomaz, D. Z. (2013). Migração haitiana para o Brasil pós-terremoto: indefinição normativa e implicações políticas. PrimeirosEstudos, (4), 131-143. Recuperado de: http://www.revistas.usp.br/ primeirosestudos/article/view/56732

Véran, J., Da Silva, D.yFainstat, T. (2014). Nem refugiados, nem migrantes: a chegada dos haitianos à cidade de Tabatinga (Amazonas). Dados. Revista de CiênciasSociais, 57(4), 1007-1041. Recuperado de http:/ /www.redalyc.org/html/218/21835768005/

\section{Fuentes:}

Brasil regulamentaconcessão de vistos para imigrantes haitianos. (12 de enero de 2012). Veja. Recuperado de https://veja.abril.com.br/mundo/brasil-regulamenta-concessao-de-vistos-para-imigrantes-haitianos/

Brasil acelerará visas para haitianos y evitar que sean víctimas de "coyotes”.(6 de febrero de 2014). Recuperado de http://hoy.com.do/brasilacelerara-visas-para-haitianos-y-evitar-que-sean-victimas-de-coyotes/ 
Comunicado de Organizaciones migrantes y promigrantes respecto al proyecto de ley de migraciones. (10 de abril de 2018). [Comunicado de prensa]. Recuperado de: http://www.revistasur.cl/revistasur.cl/2018/ 04/comunicado-de-organizaciones-migrantes-y-promigrantes-respecto-al-proyecto-de-ley-de-migraciones/

Disposición N 4362/2014.Boletín Oficial de la República Argentina, Buenos Aires, Argentina, 7 de noviembre de 2014.

Espinoza, M. (3 de mayo de 2018). Gobierno entrega balance por proceso de regularización migratoria. Diario Uchile. Recuperado de https:// radio.uchile.cl/2018/05/03/pinera-entrega-balance-por-proceso-deregularizacion-migratoria/

Ferrer Durán, C. (28 de febrero de 2018). Radiografía a la migración haitiana: Las últimas cifras, prejuicios y desafíos del fenómeno. Emol. Recuperado de https://www.emol.com/noticias/Nacional/2018/02/28/ 896852/El-exodo-haitiano-Radiografia-al-fenomeno-migratorio-quegenera-un-debate-en-torno-al-racismo-en-Chile.html

Lozano, S. (23 de agosto de 2018). Visas de turismo a los haitianos que quieren entrar al país: las razones que llevaron al Gobierno a tomar medida. Clarín. Recuperado de https://www.clarin.com/politica/visas-turismo-haitianos-quieren-entrar-pais-razones-llevaron-gobiernotomar-medida_0_rk9WYZjIQ.html

Marín, V. (10 de abril de 2018). Ministro del Interior descarta "trato discriminatorio” para haitianos tras anunciar que se les exigirá visa a quienes quieran viajar a Chile. Emol. Recuperado de https:// www.emol.com/noticias/Nacional/2018/04/10/902004/Ministro-delInterior-descarta-trato-discriminatorio-para-haitianos-tras-anunciarque-se-les-exigira-visa-a-quienes-quieran-viajar-a-Chile.html

Marcel, T. (2015). Governo brasileiro continuará emissão de visto humanitário para haitianos. Camara Noticias. Recuperado de http:/ /www2.camara.leg.br/camaranoticias/noticias/SEGURANCA/ 492508-GOVERNO-BRASILEIRO-CONTINUARA-EMISSAODE-VISTO-HUMANITARIO-PARA-HAITIANOS.html

Memorandum N 192/18. Dirección Nacional de Migraciones. Buenos Aires, Argentina, 10 de mayo de 2018.

Minuta Reforma Migratoria de Chile (Abril de 2018). Ministerio del Interior y Seguridad Pública. Recuperado de https://cdn.digital.gob.cl/ filer_public/b0/09/...2ac5.../nueva_ley_de_migracion.pdf

Resolución N 477/2018.Boletín Oficial República Argentina, Buenos Aires, Argentina, 21 de agosto de 2018. 